\title{
NODE AVERAGED NODAL INTEGRAL METHOD
}

\author{
NADEEM AHMED ${ }^{1}$, NITEEN KUMAR ${ }^{2}$ AND SUNEET SINGH ${ }^{3}$ \\ ${ }^{1}$ Department of Energy Science and Engineering \\ Indian Institute of Technology Bombay \\ nadeem.ahmed@iitb.ac.in \\ ${ }^{2}$ Department of Energy Science and Engineering \\ Indian Institute of Technology Bombay \\ krniteen@gmail.com \\ ${ }^{3}$ Department of Energy Science and Engineering \\ Indian Institute of Technology Bombay \\ suneet.singh@iitb.ac.in
}

Key words: Coarse mesh method, Nodal Integral Method, Diffusion equation

\begin{abstract}
In this paper, a new variant of the nodal integral method (NIM) is proposed for the solution of $2 \mathrm{D}$ diffusion equation. In NIM, the scheme is derived using Transverse Integration Process (TIP) and analytical solutions of ODEs within nodes. This analytical pre-processing dramatically improve the quality of the solution, which is the main distinguishing feature of NIM. However, in this new approach, the TIP is performed at node center instead of node edge. Adapting this approach simplifies the scheme and make it easier to solve. To test the capabilities of this newly developed scheme, some benchmark problems using the diffusion equation are solved. It is found that the scheme is as accurate as traditional NIM, and the scheme is relatively easy to solve. It can be said that a simplified scheme is developed while retaining the accuracy of traditional NIM.
\end{abstract}

\section{INTRODUCTION}

With the advancement of computational power, researchers have started solving more complex problems for a better understanding of the physics behind the problems. To solve any problem, first model equations are set up, which can accurately represent the system. Thereafter these model equations discretized over the domain of interest using numerical methods. A few popular numerical methods are the finite difference method (FDM), finite element method (FEM), and finite volume method (FVM), and many variants of these methods are available. However, all these methods are fine mesh methods, which required very small mesh sizes to accurately emulate the results. With higher-order schemes, these methods can be used over coarser mesh as well [1]. Although higher-order schemes need less number of grid points, their implementation is really difficult, and low order fine mesh schemes are more popular. Due to use of fine mesh, these methods result in a large system of equations, which in turn results in huge system matrices. Solving these big matrices is one of the major challenges in computational fluid dynamics. Even with such advancement in computational power, these methods need a substantial amount of computational resources and time to give acceptable results. 
Alternately, there are few coarse mesh methods available [2, 3, 4, 5], and 'Nodal Integral Method (NIM)' is one of the preferred methods. In the 70s, NIM was first developed for the solution of the neutron diffusion equation, and the accurate results encouraged its further development $[6,7]$. When NIM is compared with FDM, it was found that NIM needs four times less number of grid points for the same level of accuracy as provided by FDM [6,7]. There is an analytical pre-processing involved in the development of the NIM scheme, which drastically enhances the quality of results. In NIM, transverse integration process (TIP) and analytical pre-processing are two distinguishing features [8]. The TIP reduces the PDEs into a set of transverse averaged ODEs. Next, these sets of ODEs are solved analytically within a node. The final form of the scheme is obtained using the analytical solution of these ODEs. However, there is one constraint associated with NIM, the use of TIP restrict the scheme to regular geometries only. Some schemes based on algebraic transformations are developed to handle irregular geometries, but they are not as effective as traditional NIM [9].

In this paper, an alternate approach of Nodal Integral method for the diffusion equation is developed on the same note as developed by Shober [10] in 1976, which is capable in handling irregular geometries. One way to handel the irregular geometry is to couple the NIM with any of scheme which deals with the irregular nodes at the boundary such as cut cell approach [11]. In earlier versions of NIM variables are averaged at node edges, on the other hand in this newly proposed approach, variables are averaged at node center. In this way, node edges are free from any constraint and can be approximated to fit the geometries. This is the only key difference between traditional NIM and node averaged NIM (NANIM). However, in this work the proposed scheme is not really implemented over irregular geometries, but is expected to work well with them. Due to very simple development procedure this scheme can be imple-
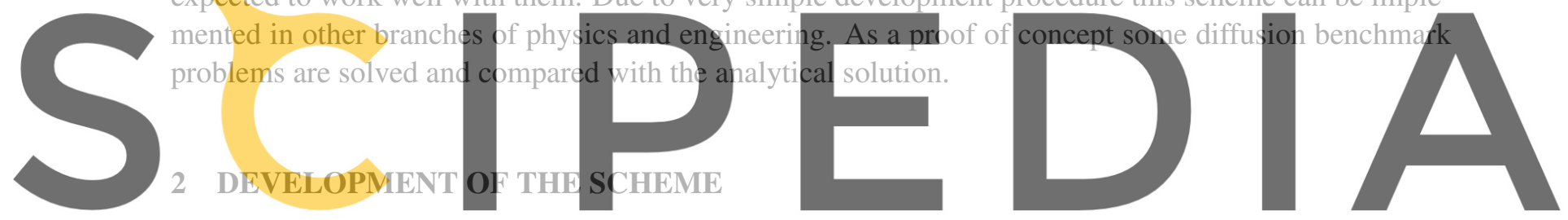

Two dimensional steady state Diffusion equation is given as

Register for free at https//www.scipedia.g̨m to download the version without the watermark

$$
\frac{\partial^{2}}{\partial x^{2}}+\frac{\partial^{2}}{\partial y^{2}}=f(x, y)
$$

After descritizing the domain, Transverse integration of Equation (1) is done by using the operator $\frac{1}{2 a_{i, j}}$ $\int_{-a_{i, j}}^{+a_{i, j}} d x$ and $\frac{1}{2 b_{i, j}} \int_{-b_{i, j}}^{+b_{i, j}} d y$, where $2 a_{i, j}$ and $2 b_{i, j}$ are the dimension of node $(i, j)$ in $x$ and $y$ direction respectively, as shown in Figure (1) [8]. The space averaged equation with respect to variable $x$ is given as

$$
\frac{1}{2 a_{i, j}} \int_{-a_{i, j}}^{+a_{i, j}} \frac{\partial^{2} T}{\partial y^{2}} d x=\frac{1}{2 a_{i, j}} \int_{-a_{i, j}}^{+a_{i, j}} f(x, y)-\frac{1}{2 a_{i, j}} \int_{-a_{i, j}}^{+a_{i, j}} \frac{\partial^{2} T}{\partial x^{2}} d x
$$

Now the R.H.S of Equation (2) is taken as a pseudo source term which is given as

$$
\frac{1}{2 a_{i, j}} \int_{-a_{i, j}}^{+a_{i, j}} \frac{\partial^{2} T}{\partial y^{2}} d x=S^{x}(y)=f^{x y}-\frac{1}{2 a_{i, j}} \int_{-a_{i, j}}^{+a_{i, j}} \frac{\partial^{2} T}{\partial x^{2}} d x
$$


where $S^{x}(y)$ is the pseudo-source term over a node $(i, j)$ and $f^{x y}$ is the averaged heat source. Now expanding the pseudo-source term (RHS of Equation (3))by Legendre polynomial and truncating at zeroth level we get the ordinary differential equation as,

$$
\frac{\partial^{2} \bar{T}^{x}}{\partial y^{2}}=\bar{S}_{1}^{x y}-\bar{f}^{x y}=\bar{S}^{x}
$$

Similarly in $y$ direction we get the second differential equation as,

$$
\frac{\partial^{2} \bar{T}^{y}}{\partial x^{2}}=\bar{S}_{2}^{y x}-\bar{f}^{y x}=\bar{S}^{y}
$$

These ODE's can also be solved analytically in the same manner as in NIM only the differnce in the present approach is that now we will add the current term before solving it for $\bar{T}^{x}$ or $\bar{T}^{y}$. For current let us integrate Equation (4) and Equation (5) with respect to $x$ and $y$, which is given as

$$
\begin{aligned}
& \frac{\partial \bar{T}^{x}}{\partial y}=\bar{S}^{x} y+C_{1}=\bar{J}^{x}(y) \\
& \frac{\partial \bar{T}^{y}}{\partial x}=\bar{S}^{y} x+D_{1}=\bar{J}^{y}(x)
\end{aligned}
$$
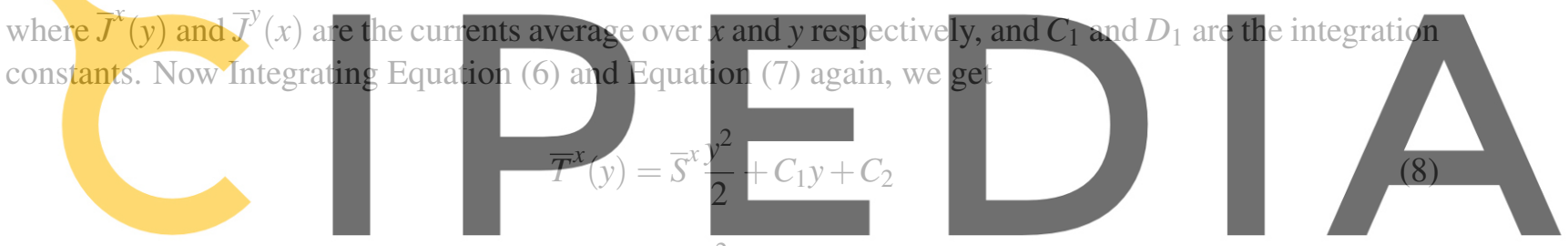

$$
\bar{T}^{y}(x)=\bar{S}^{y} \frac{x^{2}}{2}+D_{1} x+D_{2}
$$

(9) Register for free at https//www.scipedia.com to ${ }^{2}$ download the version without the watermark

We will drive expression only for the Equation (8) and the other one can be proceed in an analogus manner. Therefore for Equation (8) Boundary condition at node $(i, j)$ is shown in Figure (1) and is given as

$$
y=y_{j} \quad \bar{T}^{x}=\bar{T}_{i, j}^{x} \quad \text { and } \quad \bar{J}^{x}=\bar{J}_{i, j}^{x}
$$

By applying boundary conditions and Eliminating integrating constants $C_{1}$ and $C_{2}$ from Equation (8) we get

$$
\bar{T}^{x}(y)=\bar{T}_{i, j}^{x}+\frac{1}{2} \bar{S}_{i, j}^{x}\left(y-y_{j}\right)^{2}+\bar{J}_{i, j}^{x}\left(y-y_{j}\right)
$$

Similarly applying the boundary condition at node $(i, j-1)$ and eliminating $C_{1}$ and $C_{2}$ form (8) we get

$$
\bar{T}^{x}(y)=\bar{T}_{i, j}^{x}+\frac{1}{2} \bar{S}_{i, j-1}^{x}\left(y_{j}-y\right)^{2}+\bar{J}_{i, j}^{x}\left(y_{j}-y\right)
$$




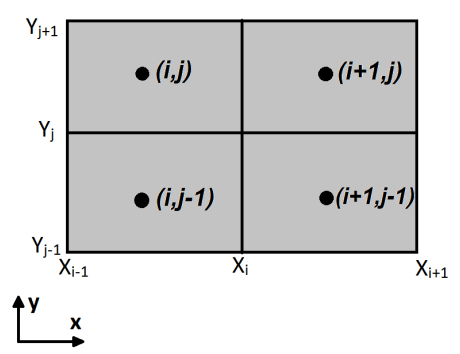

Figure 1: Stencil for Node averaged Nodal method

Operating the operator $\frac{1}{y_{j+1}-y_{j}} \int_{y_{j}}^{y_{j+1}} d y$ in Equation (10) and $\frac{1}{y_{j}-y_{j-1}} \int_{y_{j}}^{y_{j-1}} d y$ in Equation (11), we get the equations in node average variables [10] which is given as

$$
\begin{aligned}
\bar{T}_{i, j}^{x y} & =\frac{1}{6}\left(6 \bar{T}_{i, j}^{x}+\left(-3 \bar{J}_{i, j}^{x}+\bar{S}_{i, j}^{x}\left(y_{j}-y_{j+1}\right)\right)\left(y_{j}-y_{j+1}\right)\right) \\
\bar{T}_{i, j-1}^{x y} & =\frac{1}{6}\left(6 \bar{T}_{i, j}^{x}+\left(-3 \bar{J}_{i, j}^{x}+\bar{S}_{i, j-1}^{x}\left(y_{j}-y_{j-1}\right)\right)\left(y_{j}-y_{j-1}\right)\right)
\end{aligned}
$$

Now eliminating the surface average term i.e. $T_{i, j}^{y}$ from Equation (12) and Equation (13) and solving for

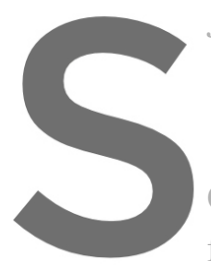
$J_{i, j}^{x}$ as
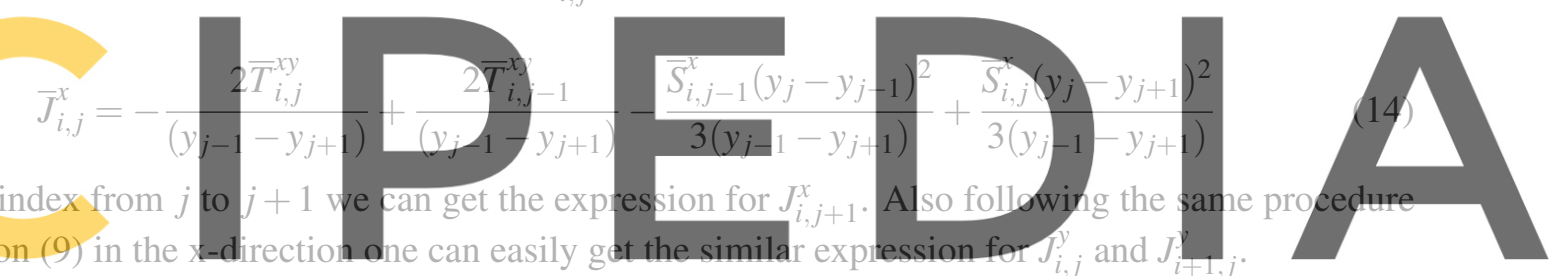

Constraint equation are required for the final formulation of the scheme [8]. The first constraint equation

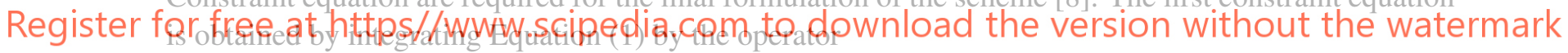

$\frac{1}{4\left(x_{i+1}-x_{i}\right)\left(y_{i+1}-y_{j}\right)} \int_{x_{i}}^{x_{i+1}} \int_{y_{j}}^{y_{j+1}} d y d x$ that will lead to the relation between $\bar{S}_{i, j}^{x}$ and $\bar{S}_{i, j}^{y}$ and that is,

$$
\bar{S}_{i, j}^{x}+\bar{S}_{i, j}^{y}=\bar{f}_{i, j}^{x y}
$$

The other two constraint equation we will get by doing some approxiamtions in pseudo-source terms [10] which gives us the following relations

$$
\begin{aligned}
& \bar{S}_{i, j}^{x}=\bar{f}_{i, j}^{x y}-\frac{\left(\bar{J}_{i+1, j}^{y}-\bar{J}_{i, j}^{y}\right)}{\left(x_{i+1}-x_{i}\right)} \\
& \bar{S}_{i, j}^{y}=\bar{f}_{i, j}^{x y}-\frac{\left(\bar{J}_{i, j+1}^{x}-\bar{J}_{i, j}^{x}\right)}{\left(y_{i+1}-y_{i}\right)}
\end{aligned}
$$

Now putting the expressions of currents as given in Equation (14) from both directions in both the 
Equations (16) and (17) we get

$$
\begin{aligned}
& \bar{S}_{i, j}^{y}=\bar{f}_{i, j}^{x y}-\frac{\bar{T}_{i, j-1}^{x y}}{h^{2}}+\frac{2 \bar{T}_{i, j}^{x y}}{h^{2}}-\frac{\bar{T}_{i, j+1}^{x y}}{h^{2}}+\frac{\bar{S}_{i, j-1}^{x}}{6}-\frac{\bar{S}_{i, j}^{x}}{3}+\frac{\bar{S}_{i, j+1}^{x}}{6} \\
& \bar{S}_{i, j}^{x}=\bar{f}_{i, j}^{x y}-\frac{\bar{T}_{i-1, j}^{x y}}{h^{2}}+\frac{2 \bar{T}_{i, j}^{x y}}{h^{2}}-\frac{\bar{T}_{i+1, j}^{x y}}{h^{2}}+\frac{\bar{S}_{i-1, j}^{y}}{6}-\frac{\bar{S}_{i, j}^{y}}{3}+\frac{\bar{S}_{i+1, j}^{y}}{6}
\end{aligned}
$$

Now putting $S_{i, j}^{x}$ and $S_{i, j}^{y}$ in Equation (15) we get the final equation for Node average temperature

$$
\begin{gathered}
\bar{T}_{i-1, j}^{x y}=\frac{1}{24}\left(2 \bar{S}_{i, j}^{x}+2 \bar{S}_{i, j}^{y}-\bar{S}_{i, j-1}^{x}-\bar{S}_{i, j+1}^{x}-\bar{S}_{i-1, j}^{y}-\bar{S}_{i+1, j}^{y}-6 \bar{f}_{i, j}^{x y}\right) \\
+\frac{1}{4}\left(\bar{T}_{i-1, j}^{x y}+\bar{T}_{i+1, j}^{x y}+\bar{T}_{i, j-1}^{x y}+\bar{T}_{i, j+1}^{x y}\right)
\end{gathered}
$$

Now we have three equations and three unknowns, we can solve this by any of the standard numerical solvers.

\section{RESULTS AND DISCUSSION}

Based on the numerical scheme developed in the previous section standard problem has been numerically solved and compared with their analytical solution

the space for comparison

get the best match. As

problems are compared wit

Numerical verification
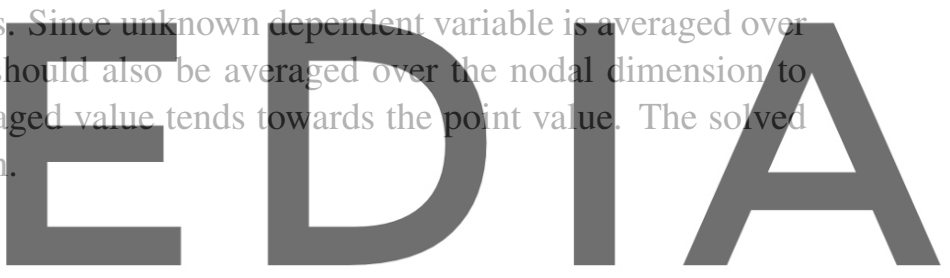

Problem 1:

Register for free at https//www.scipedia.com to download the version without the watermark

Problem is taken from [12] which is to find the solution of Poisson's equation $\Delta^{2} T=-2$ in square with

boundary conditions $T(0, y)=0, T(L, y)=0, T(x, 0)=0, T(x, L)=0$. The analytical solution of the

above equation is,

$$
T(x, y)=\frac{32 L^{2}}{\pi^{4}} \sum_{m, n, o d d} \frac{1}{m n\left(m^{2}+n^{2}\right)} \sin \left(\frac{n \pi x}{L}\right) \cos \left(\frac{m \pi y}{L}\right)
$$

Where ' $n$ ' is the no. of nodes in $x$-direction and ' $m$ ' is no. of nodes in $y$-direction. 


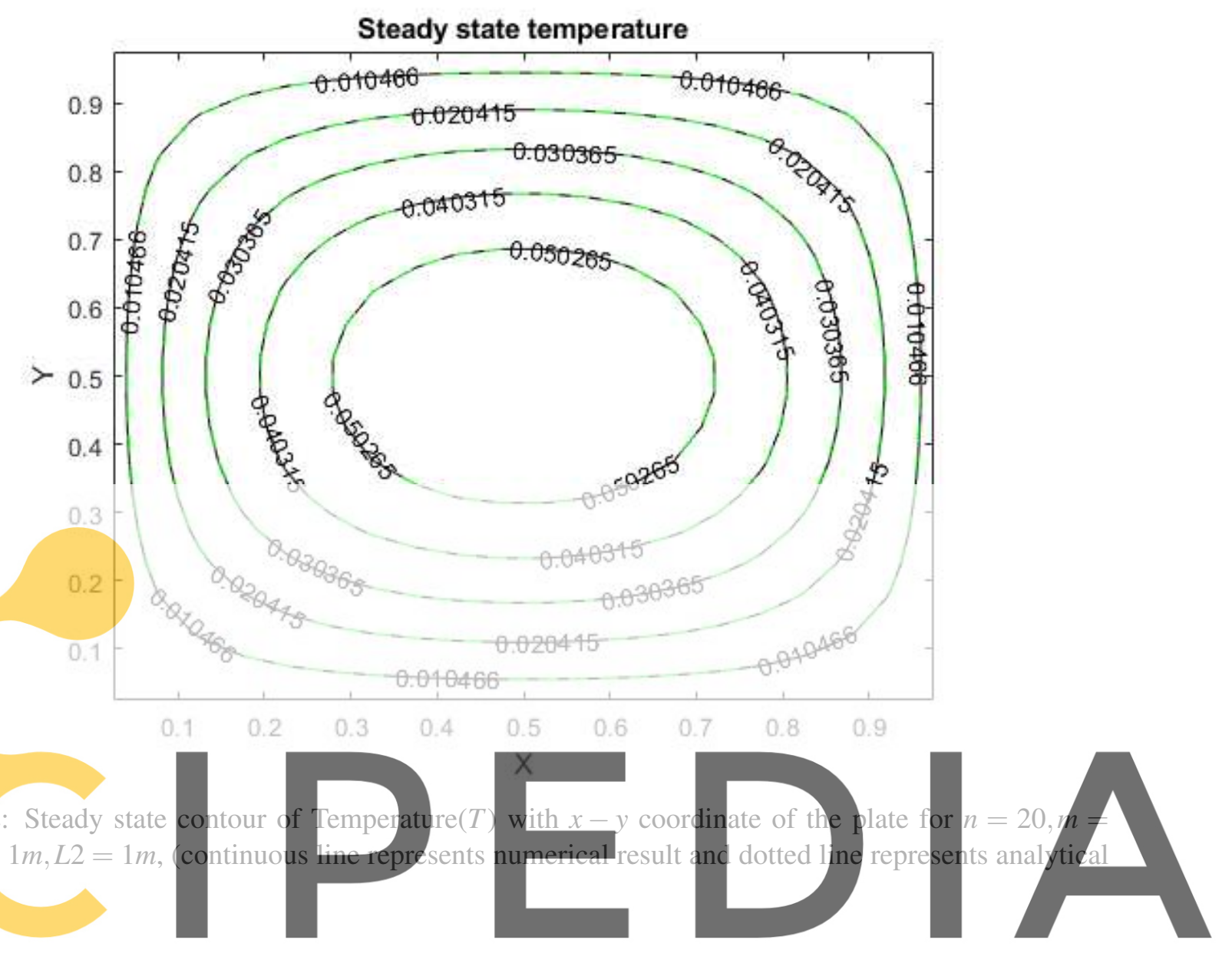

Problem 2:

Register for free at https//www.scipedia.com to download the version without the watermark

Problem is taken from [12] to Solve for the steady state temperature distribution in square plate $(0<$

$x<L) ;(0<y<L)$, whose all sides are kept at zero temperature and there is volumetric heat source

in the plate given as $\sin \left(\frac{\pi x}{L}\right)$. Thus solve Poisson equation $\Delta^{2} T=-\sin \left(\frac{\pi x}{L}\right)$ with boundary condition $T=0(x=0, x=L, y=0, y=L)$ and compare with the analytical solution.

The analytical solution is given as

$$
T(x, y)=\left(\frac{L}{\pi^{2}}\right) \sin \left(\frac{\pi x}{L}\right)\left\{1-\cosh \left(\frac{\pi y}{L}\right)+\left[\frac{\cosh (\pi)-1}{\sinh (\pi)}\right] \sinh \left(\frac{\pi y}{L}\right)\right\}
$$

It is clear from Figure (3) that the numerical result is exactly matching with each other the reason behind this the shape function that is derived from the solution of differential equation of averaged temperature in one dimension in a node is quadratic in nature and that is close to the trigonometric variation of analytical solution. 


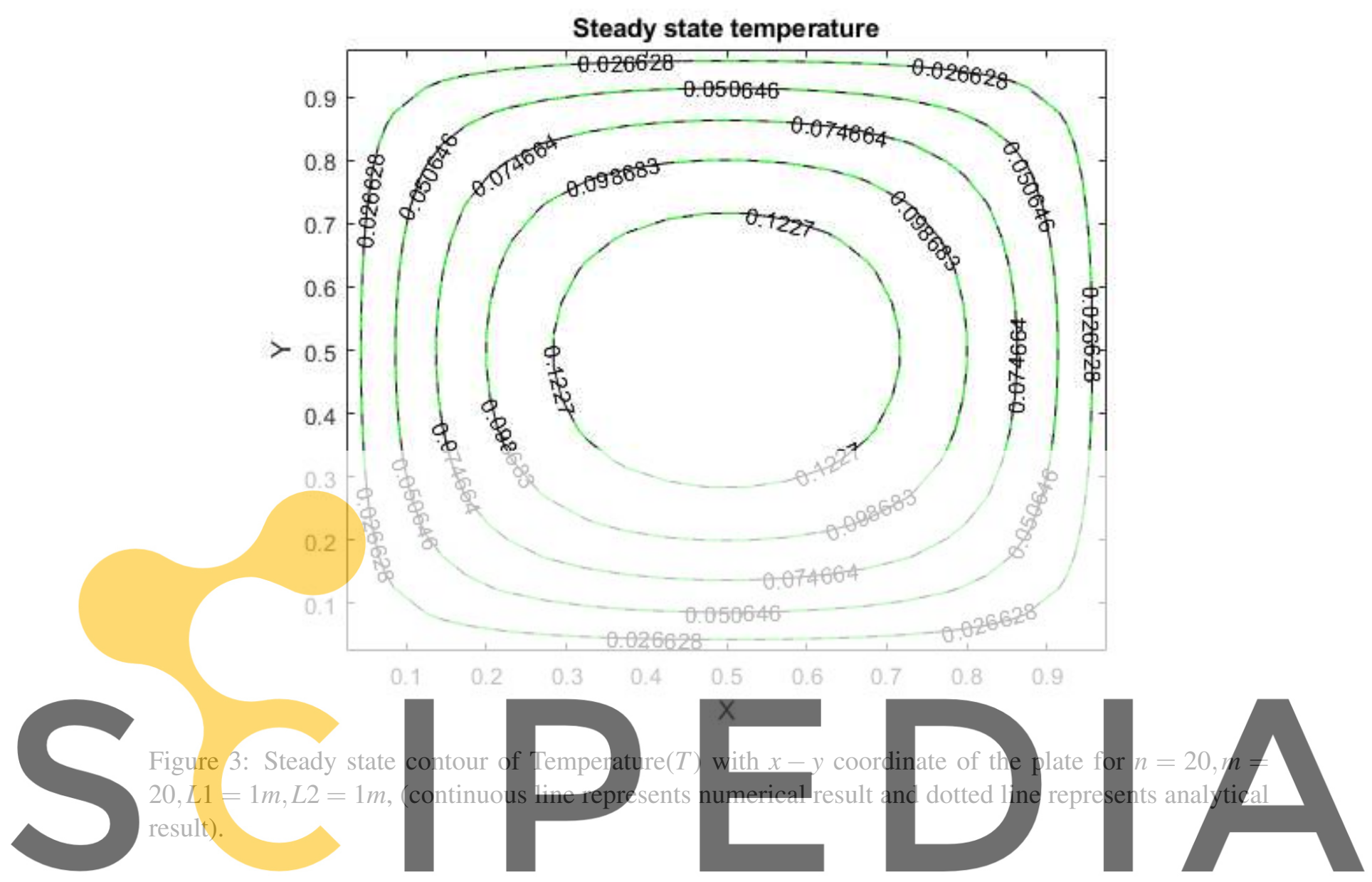

4 CONCLUSIONS

Register for free at https//www.scipedia.com to download the version without the watermark - A new approach of nodal method is developed which deals with the node averaged variable instead of surface averaged variable as done in traditional NIM. The accuracy of the purposed algorithm is almost simillar to the traditional NIM. Two dimensional diffusion equation is taken for the formulation of the scheme and the iterative method is used for the numerical solution. Some benchmark problems of diffusion equation are solved to check the applicability of the scheme and compared the numerical results with the analytical solution and it is found that this scheme hold the same accuracy in coarser grid as in traditional NIM.

- The scheme can easily be extended for further fluid flow problem such as convection-diffusion equation, Burgers' equation and Navier-Stokes equation. On the other hand one can easily couple this scheme with other scheme which deals with the cell centroids such as cut cell approach and the limitation of NIM can be overcome. 


\section{REFERENCES}

[1] Rizwan-uddin. An Improved Coarse-Mesh nodal Integral Method for Partial Differential Equations. Numerical Methods for Partial Differential Equations (1997) 13:113-147.

[2] Azmy, Y.Y. and Dorning, J.J. A nodal integral approach to the numerical solution of partial differential equations. Advances in Reactor Computations (1983) 2:893-909.

[3] William, C.H. and Dorning, J.J. Nodal coarse-mesh method for the efficient numerical solution of laminar flow problems. Journal of Computational Physics (1985) 59:405-440.

[4] Michael, E.P.E. Dorning, J.J. and Rizwan-uddin. Studies on Nodal Integral Methods for the Convection-Diffusion Equation. Nuclear Science and Engineering (2001) 137:380-399.

[5] Zhou, X. Guo, J. Li, F. General nodal expansion method for multi-dimensional steady and transient convection-diffusion equation. Annals of Nuclear Energy (2016) 88:118-125.

[6] Lawrence, R.D. Progress in nodal methods for the solution of the neutron diffusion and transport equations. Progress in Nuclear Energy (1986) 17:271-301.

[7] Azmy, Y.Y. Nodal Method for Problems in Fluid Mechanics and Neutron Transport. University of Illinois At Urbana-Champaign, (1985).

[8] Rizwan-uddin. A second-order space and time nodal method for the one-dimensional convectiondiffusion equation. Computers \& Fluids (1997) 26:233-247.

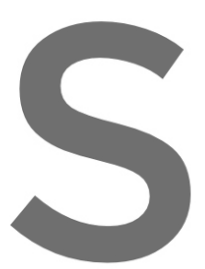

[9] Neeraj, K. Suneet, Transport Equations 64:1-21. Annals of 1

[10] Shober, R.A. Nonlin Institute of Technolo
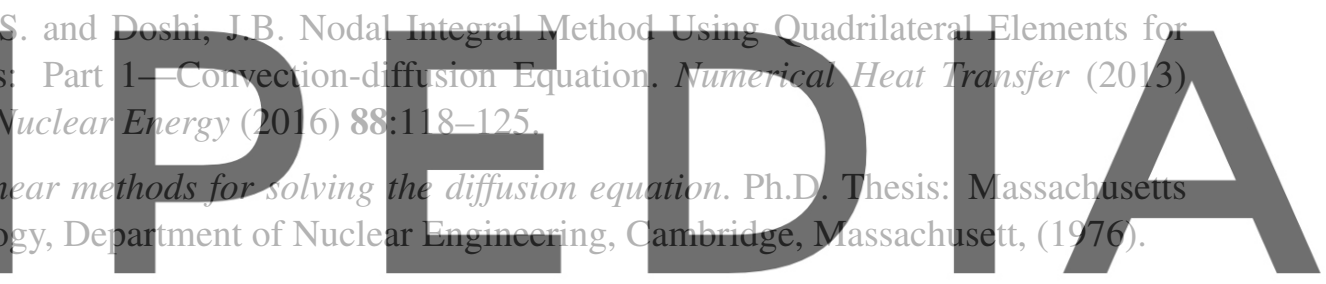

[11] Hua, J. Fue-Sang, L. and Eugene, Y. An efficient second-order accurate cut-cell method for solving

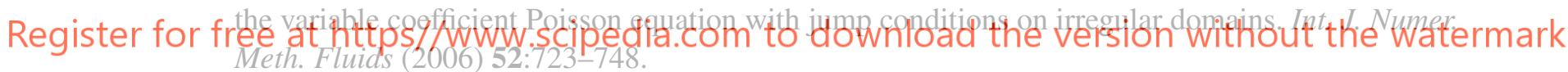

[12] Doshi J.B. Differential Equations for Scientists and Engineers. Narosa Publishing House, New Delhi, (2010). 